\title{
Stability Analysis of a Population Model with Maturation Delay and Ricker Birth Function
}

\author{
Chongwu Zheng, ${ }^{1}$ Fengqin Zhang, ${ }^{1}$ and Jianquan $\mathrm{Li}^{2}$ \\ ${ }^{1}$ Department of Mathematics, Yuncheng University, Shanxi, Yuncheng 044000, China \\ ${ }^{2}$ Science College, Air Force Engineering University, Xian 710051, China \\ Correspondence should be addressed to Jianquan Li; jianq_li@263.net
}

Received 29 September 2013; Accepted 19 March 2014; Published 16 April 2014

Academic Editor: Robert Szalai

Copyright ( 12014 Chongwu Zheng et al. This is an open access article distributed under the Creative Commons Attribution License, which permits unrestricted use, distribution, and reproduction in any medium, provided the original work is properly cited.

\begin{abstract}
A single species population model is investigated, where the discrete maturation delay and the Ricker birth function are incorporated. The threshold determining the global stability of the trivial equilibrium and the existence of the positive equilibrium is obtained. The necessary and sufficient conditions ensuring the local asymptotical stability of the positive equilibrium are given by applying the Pontryagin's method. The effect of all the parameter values on the local stability of the positive equilibrium is analyzed. The obtained results show the existence of stability switch and provide a method of computing maturation times at which the stability switch occurs. Numerical simulations illustrate that chaos may occur for the model, and the associated parameter bifurcation diagrams are given for certain values of the parameters.
\end{abstract}

\section{Introduction}

The model of a single species population growth is usually the base of modeling transmission of some infection and interaction between two or more species. Cooke et al. [1] proposed the model of a single species population model

$$
\frac{d N}{d t}=b e^{-d_{1} \tau} e^{-a N(t-\tau)} N(t-\tau)-d N(t) .
$$

Here $N=N(t)$ denotes the mature population of the species; $d_{1}$ and $d$ are the death rates of the immature and mature population, respectively; the delay $\tau$ is the maturation time, and $e^{-d_{1} \tau}$ is the probability in which an immature individual keeps surviving to mature; the Ricker function $b e^{-a N}$ represents the per capita birth rate of mature individuals, which reflects the dependence of population birth on the density of individuals, and the parameter $b$ is the per capita maximal birth rate. Model (1) has been applied to describe some epidemiological and population biological models [1-5].

For model (1), Cooke et al. [1] found the existence of stability switch as $\tau$ increases, by using the geometric method. Jiang and Zhang [6] theoretically discussed the stability switch of model (1) by means of the geometric criterion proposed by Beretta and Kuang [7], where $\tau$ is used as the bifurcation parameter. Wei and Zou [8] considered the local and global Hopf bifurcation for (1), where $b$ is used as the bifurcation parameter.

In this paper, our aim is to investigate the stability of model (1) and completely analyze the effect of all the parameter values on the stability. By making the suitable scaling, model (1) is reduced, and the necessary and sufficient conditions for the stability of the positive equilibrium of the simplified model are obtained. The stability switch is also proved theoretically, and the associated conditions are given. The obtained results supplement the conclusions in $[1,6,8]$, and numerical simulations illustrate the existence of chaos for certain parameter values. The associated parameter bifurcation diagrams are plotted for certain values of the parameters.

The paper is organized as follows. In the next section, model (1) is reduced, and the stability is analyzed by the LaSalle's invariance principle and the Pontryagin's method. In Section 3 , the effect of all the parameter values on the stability of the positive equilibrium is discussed, and the associated 
parameter bifurcation diagrams are given for certain values of the parameters to show the complexity of dynamical behaviors of the model. Finally, a brief conclusion is given.

\section{Stability Analysis}

For (1), making the scaling

$$
\bar{N}(t)=a N(t), \quad \bar{t}=d t, \quad \bar{\tau}=d \tau,
$$

and denoting $\bar{b}=b / d$, then dropping their bars yields

$$
\frac{d}{d t} N\left(\frac{t}{d}\right)=b e^{-d_{1} \tau} e^{-N((t-\tau) / d)} N\left(\frac{t-\tau}{d}\right)-N\left(\frac{t}{d}\right) .
$$

Denote $N(t / d)$ still by $N(t)$, then (3) becomes

$$
\frac{d N}{d t}=b e^{-d_{1} \tau} e^{-N(t-\tau)} N(t-\tau)-N(t)
$$

From the biological meaning, the initial conditions for (4) are given as follows:

$$
N(\theta)=\phi(\theta), \quad \theta \in[-\tau, 0],
$$

where $\phi(\theta) \geq 0$ and $\phi(0)>0$.

The following theorem establishes the positivity and boundedness of solutions of (4).

Theorem 1. All solutions of (4) under the initial condition (5) are positive on $[0,+\infty)$ and ultimately bounded.

Proof. Assume that there is $t_{1}\left(t_{1}>0\right)$ such that $N\left(t_{1}\right)=0$; then it follows from $N(0)>0$ and the continuity of solution of (4) that there is $t^{*}=\inf \{t: t>0, N(t)=0\}$ such that $N(t)>0$ for $t \in\left[0, t^{*}\right)$. So we have $N^{\prime}\left(t^{*}\right) \leq 0$. However, $N^{\prime}\left(t^{*}\right)=b e^{-d_{1} \tau} N\left(t^{*}-\tau\right) e^{-N\left(t^{*}-\tau\right)}>0$. This contradiction implies that $N(t)>0$ for $t>0$. Therefore, all solutions of (4) under the initial condition (5) are positive on $[0,+\infty)$.

On the other hand, we know that $x e^{-x} \leq e^{-1}$ for $x>0$; then, under the initial condition (5), from (4) we have $N^{\prime} \leq$ $b e^{-1-d_{1} \tau}-N$. It follows that $\limsup _{t \rightarrow+\infty} N(t) \leq b e^{-1-d_{1} \tau}$; that is, all solutions of (4) under the initial condition (5) are ultimately bounded, and the set $D=\left\{N(t) \in C_{+}: 0 \leq N(t) \leq\right.$ $\left.b e^{-1-d_{1} \tau}\right\}$ is positively invariant for (4).

The proof of Theorem 1 is complete.

Obviously, (4) always has the trivial equilibrium $N=0$, and, when $b e^{-d_{1} \tau}>1$, that is, $\ln b-d_{1} \tau>0$, (4) also has a unique positive equilibrium $N^{*}=\ln b-d_{1} \tau$, where $N^{*} \in D$ since $N^{*} \leq b e^{-1-d_{1} \tau}$. Notice that $b e^{-d_{1} \tau}>1$ implies that $b>1$.

With respect to the stability of the trivial equilibrium $N=$ 0 , we have the following statement.

Theorem 2. The trivial equilibrium $N=0$ of (4) is globally stable as be $e^{-d_{1} \tau} \leq 1$ and unstable as $b e^{-d_{1} \tau}>1$.

Proof. Define a Lyapunov functional $L=N+$ $b e^{-d_{1} \tau} \int_{t-\tau}^{t} N(\theta) e^{-N(\theta)} d \theta$, then the derivative of $L$ with respect to $t$ along solutions of (4) is given by

$$
\frac{d L}{d t}=b e^{-d_{1} \tau} N e^{-N}-N .
$$

For $N \geq 0$,

$$
\frac{d L}{d t}=b e^{-d_{1} \tau} N e^{-N}-N \leq\left(b e^{-d_{1} \tau}-1\right) N .
$$

When $b e^{-d_{1} \tau} \leq 1, d L / d t \leq 0$ and the equality holds if and only if $N=0$. It is easy to know that the largest invariant set of (4) on the set $\left\{\left(N(t) \in D: L^{\prime}(t)=0\right)\right\}$ is the singleton $\{O\}$. It follows by the LaSalle's invariance principle [9] that the trivial equilibrium $O$ of (4) is globally stable on the set $D$ as $b e^{-d_{1} \tau} \leq 1$.

The characteristic equation of (4) at $N=0$ is given by

$$
\Phi(\lambda):=\lambda+1-b e^{-d_{1} \tau} e^{-\lambda \tau}=0 .
$$

Since $\Phi(0)=1-b e^{-d_{1} \tau}<0$ for $b e^{-d_{1} \tau}>1$, and $\lim _{\lambda \rightarrow+\infty} \Phi(\lambda)=+\infty$, equation $\Phi(\lambda)=0$ must have positive root as $b e^{-d_{1} \tau}>1$. Therefore, the trivial equilibrium $N=0$ of (4) is unstable as $b e^{-d_{1} \tau}>1$.

The proof of Theorem 2 is complete.

Remark 3. From biological meaning, the global stability of the trivial equilibrium implies the eventual extinction of the population, and its instability implies the persistence of the population. Therefore, the population is extinct finally if $b e^{-d_{1} \tau} \leq 1$ and keeps survival if $b e^{-d_{1} \tau}>1$.

In the following we consider the stability of the positive equilibrium $N=N^{*}$. The linearized equation of (4) at $N=$ $N^{*}$ is given by

$$
\frac{d x}{d t}=\left(1+d_{1} \tau-\ln b\right) x(t-\tau)-x(t) .
$$

Substituting $x=c e^{\lambda t}$ with $c \neq 0$ into (9), we get the characteristic equation

$$
\lambda+1-\left(1+d_{1} \tau-\ln b\right) e^{-\lambda \tau}=0 .
$$

Obviously, the root of (10) with $\tau=0$ is $\lambda=-\ln b<0$ for $b>1$; that is, the positive equilibrium is locally asymptotically stable as $\tau=0$ and $b>1$. Therefore, for $b>1$, with variation of $\tau$ stability of the positive equilibrium $N^{*}$ can change only when the pure imaginary roots of (10) appear.

For the stability of $N=N^{*}$, analysis is realized by considering two cases: $0<\ln b-d_{1} \tau \leq 2$ and $\ln b-d_{1} \tau>2$. For the case $0<\operatorname{lnb}-d_{1} \tau \leq 2$, we have the following statement.

Theorem 4. The positive equilibrium $N^{*}$ of (4) is locally asymptotically stable if $0<\ln b-d_{1} \tau \leq 2$.

Proof. Suppose that there is a root of (10) with nonnegative real part if $0<\ln b-d_{1} \tau<2$, then denote the root by $\lambda=$ $\alpha+i \beta$, where $\alpha \geq 0$. Substituting it into (10) gives

$$
(\alpha+1)+i \beta=\left(1+d_{1} \tau-\ln b\right) e^{-\tau(\alpha+i \beta)} .
$$

Note that $0<\ln b-d_{1} \tau<2$ is equivalent to $\left|1+d_{1} \tau-\ln b\right|<$ 1 ; then, for $\alpha \geq 0$, the norm of the left-hand side of (11) is not less than one, but the norm of the right-hand side is less 
than one. So the contradiction occurs; that is, the assumption does not hold. Therefore, all roots of (10) are with negative real parts as $0<\ln b-d_{1} \tau<2$.

When $\ln b-d_{1} \tau=2$, (10) becomes

$$
\lambda+1+e^{\lambda \tau}=0
$$

Note that $\lambda=0$ and any pure imaginary number are not the root of (12) and that (12) with $\tau=0$ only has the root $\lambda=-1$; then all roots of (12) are with negative real parts.

Summarizing the above inferences, the positive equilibrium $N^{*}$ is locally asymptotically stable as $0<\ln b-d_{1} \tau \leq 2$.

This completes the proof of Theorem 4 .

In the following we consider the case $\ln b-d_{1} \tau>2$; that is, $1+d_{1} \tau-\ln b<-1$, by applying the Pontryagin's method [10], which is introduced in the appendix.

Let $\rho=\lambda \tau$, then (10) can become

$$
P\left(\rho, e^{\rho}\right):=\rho e^{\rho}+\tau e^{\rho}-\tau\left(1+d_{1} \tau-\ln b\right)=0 .
$$

Separating the real and imaginary parts of $P\left(i \omega, e^{i \omega}\right)$ gives

$$
P\left(i \omega, e^{i \omega}\right)=F(\omega)+i G(\omega)
$$

where

$$
\begin{aligned}
& F(\omega)=-\omega \sin \omega+\tau\left[\cos \omega-\left(1+d_{1} \tau-\ln b\right)\right] \\
& G(\omega)=\omega \cos \omega+\tau \sin \omega
\end{aligned}
$$

According to the Pontryagin's method, we first discuss zeros of $G(\omega)$ and then consider position of the roots of (13) on the complex plane. With respect to zeros of $G(\omega)$ we have the following statement.

\section{Proposition 5. All zeros of $G(\omega)$ are real.}

Proof. If $g(\omega, u, v)=\omega u+\tau v$, then $G(\omega)=g(\omega, \cos \omega$, $\sin \omega)$ and the function $\Phi_{*}^{(s)}(\omega)$ in Theorem 10 in the appendix is $\cos \omega$. Therefore, we may take $\varepsilon=0$ in Theorem 10. From Theorem 10, all zeros of $G(\omega)$ are real if and only if there are $4 k+1$ real zeros of $G(\omega)$ in the interval $[-2 k \pi, 2 k \pi]$ for $k$ a sufficiently large integer. Observe that $G(0)=0$ and that $\omega=n \pi(n= \pm 1, \pm 2, \ldots)$ is not zero of $G(\omega)$; then, for $\omega \neq 0$, $G(\omega)=0$ is equivalent to the equation

$$
-\frac{\tau}{\omega}=\cot \omega
$$

According to the graphics of functions $-\tau / \omega$ and $\tan \omega$ (Figure 1), it is easy to see that (16) has $2 k$ roots in the interval $(0,2 k \pi)$, denoted by $\omega_{n}, n=1,2,3, \ldots, 2 k$, and $\omega_{n} \in$ $((n-1 / 2) \pi, n \pi)$. Since both functions $-\gamma / \omega$ and $\tan \omega$ are odd functions, (16) also has $2 k$ roots in the interval $(2 k \pi, 0)$, denoted by $\omega_{-n}, n=1,2,3, \ldots, 2 k$, and $\omega_{-n}=-\omega_{n} \epsilon$ $(-n \pi,-(n-1 / 2) \pi)$. Thus, together with the zero of $G(\omega)$, $\omega=0, G(\omega)$ has exactly $4 k+1$ zeros in the interval $[-2 k \pi, 2 k \pi]$ for $k$ any positive integer. It follows from Theorem 10 that Proposition 5 is true.

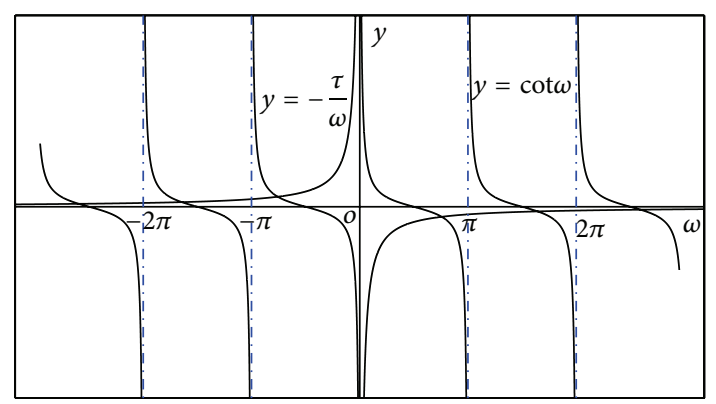

Figure 1: The figures of functions $y=-\tau / \omega$ and $y=\cot \omega$.

Theorem 9 in Appendix gives the necessary and sufficient conditions ensuring that all roots of (13) have negative real parts. So, by applying Theorem 9, we may get the necessary and sufficient conditions ensuring that $N=N^{*}$ is locally asymptotically stable. The corresponding result is as follows.

Theorem 6. When $\ln b-d_{1} \tau>2$, the positive equilibrium $N^{*}$ of (4) is locally asymptotically stable if and only if $1-\left(1+d_{1} \tau-\right.$ $\ln b) \cos \omega_{1}>0$, where $\omega_{1}$ is the zero of function $G(\omega)$ in the interval $(\pi / 2, \pi)$.

Proof. Proposition 5 has shown that all zeros of $G(\omega)$ are real, then according to Theorem 9, we only need to verify that $G^{\prime}(\omega) F(\omega)>0$ holds for all zeros of $G(\omega)$.

Since

$$
G(\omega)=\omega \sin \omega\left(\cot \omega+\frac{\tau}{\omega}\right) \text { for } \omega \neq 0
$$

for each root $\omega^{*}$ of (16) we have

$$
G^{\prime}\left(\omega^{*}\right)=-\omega^{*} \sin \omega^{*}\left(\csc ^{2} \omega^{*}+\frac{\tau}{\omega^{* 2}}\right) .
$$

Then, according to the position of zeros of $G(\omega)$, we have $G^{\prime}\left(\omega_{ \pm n}\right)<0$ for $n=1,3,5, \ldots$, and $G^{\prime}\left(\omega_{ \pm n}\right)>0$ for $n=$ $2,4,6, \ldots$

Note that the inequality $\ln b-d_{1} \tau>2$ is equivalent to the inequality $1+d_{1} \tau-\ln b<-1$, then $\cos \omega-\left[1+d_{1} \tau-\ln b\right]>0$. So $F\left(\omega_{ \pm n}\right)>0$ for $n=2,4,6, \ldots$. Therefor we have

$$
G^{\prime}\left(\omega_{ \pm n}\right) F\left(\omega_{ \pm n}\right)>0 \quad \text { for } n=2,4,6, \ldots
$$

From $G(\omega)=0$ we get $\omega=-\tau \tan \omega$. Substituting it into $F(\omega)$ gives

$$
F(\omega)=\tau \frac{1-\left(1+d_{1} \tau-\ln b\right) \cos \omega}{\cos \omega} .
$$

Since $\omega_{-n}=-\omega_{n}$ and $F(\omega)$ is an even function of $\omega$, we will only prove that $F\left(\omega_{2 n+1}\right)<0$ for $n=0,1,2,3, \ldots$, as $1-(1+$ $\left.d_{1} \tau-\ln b\right) \cos \omega_{1}>0$.

Let $\omega_{2 n+1}=(2 n+1) \pi-\bar{\omega}_{2 n+1}$, then $\bar{\omega}_{2 n+1} \in(0, \pi / 2)$, and it is easy to see that $\bar{\omega}_{2 n+1}$ is increasing with increasing of $n$. And

$$
\begin{aligned}
F\left(\omega_{2 n+1}\right) & =\tau \frac{1-\left(1+d_{1} \tau-\ln b\right) \cos \omega_{2 n+1}}{\cos \omega_{2 n+1}} \\
& =-\tau \frac{1+\left(1+d_{1} \tau-\ln b\right) \cos \bar{\omega}_{2 n+1}}{\cos \bar{\omega}_{2 n+1}} .
\end{aligned}
$$




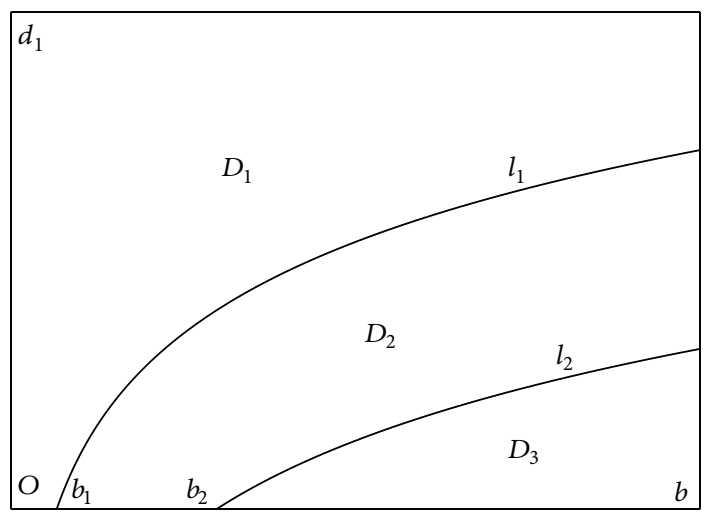

FIGURE 2: The regions corresponding to stability of equilibria $N=0$ and $N=N^{*}$ for the given $\tau$, where $l_{1}$ represents the curve $d_{1}=$ $\ln b / \tau, l_{2}$ represents the curve $d_{1}=\left(\ln b-1+\sec \omega_{1}\right) / \tau, b_{1}=1$, and $b_{2}=\exp \left(1-\sec \omega_{1}\right)$.

By monotonicity of $\bar{\omega}_{2 n+1}$ with respect to $n, 1+\left(1+d_{1} \tau-\right.$ $\ln b) \cos \bar{\omega}_{2 n+1}$ is increasing as $n$ increases. So $1+\left(1+d_{1} \tau-\right.$ $\ln b) \cos \bar{\omega}_{1}>0$ implies that $1+\left(1+d_{1} \tau-\ln b\right) \cos \bar{\omega}_{2 n+1}>$ 0 ; that is, $F\left(\omega_{2 n+1}\right)<0$. Since $1+\left(1+d_{1} \tau-\ln b\right) \cos \bar{\omega}_{1}=$ $1-\left(1+d_{1} \tau-\ln b\right) \cos \omega_{1}$, we get $G^{\prime}\left(\omega_{2 n+1}\right) F\left(\omega_{2 n+1}\right)>0$ for $n=0,1,2, \ldots$, when $1-\left(1+d_{1} \tau-\ln b\right) \cos \omega_{1}>0$.

The proof of Theorem 6 is complete.

\section{Dependence of Stability of Equilibria on the Values of Parameters}

In the previous section, we have analyzed the stability of equilibria of (4). In this section, we will investigate the dependence of stability of equilibria on the values of all parameters.

For an arbitrary positive number $\tau,(16)$ has a unique root $\omega$ in the interval $(\pi / 2, \pi)$. This root is only determined by the value of $\tau$ and is independent of the parameters $b$ and $d_{1}$. Then, for a given positive number $\tau$, the conditions in Theorem 6 can be expressed in the inequalities $\ln b-1+$ $\sec \omega_{1}<d_{1} \tau<\ln b-2$. Combining the condition in Theorem 4 , the positive equilibrium $N=N^{*}$ of (4) is locally asymptotically stable if and only if $\ln b-1+\sec \omega_{1}<d_{1} \tau<\ln b$ for $b>1$ and $d_{1}>0$ and unstable if $d_{1} \tau<\ln b-1+\sec \omega_{1}$ for $b>\exp \left(1-\sec \omega_{1}\right)$ and $d_{1}>0$. Further, according to Theorems 2, 4, and 6, for an arbitrary given positive number $\tau$, we can determine the stability of equilibria $N=0$ and $N=N^{*}$ of (4) in the associated region in the $b-d_{1}$ plane (Figure 2). That is, denote

$$
\begin{aligned}
D_{1}= & \left\{\left(b, d_{1}\right): b>0, d_{1}>0, d_{1} \tau \geq \ln b\right\}, \\
D_{2}= & \left\{\left(b, d_{1}\right): b>1, d_{1}>0,\right. \\
& \left.\ln b-1+\sec \omega_{1}<d_{1} \tau<\ln b\right\}, \\
D_{3}= & \left\{\left(b, d_{1}\right): b>\exp \left(1-\sec \omega_{1}\right), d_{1}>0,\right. \\
& \left.d_{1} \tau<\ln b-1+\sec \omega_{1}\right\} ;
\end{aligned}
$$

then, for the given $\tau$, when $\left(b, d_{1}\right) \in D_{1}, N=0$ is globally stable; when $\left(b, d_{1}\right) \in D_{2}, N=N^{*}$ is locally asymptotically stable, and $N=0$ is unstable; when $\left(b, d_{1}\right) \in D_{3}$, both $N=$ 0 and $N=N^{*}$ are unstable. Biologically, the population is extinct eventually as $\left(b, d_{1}\right) \in D_{1}$ and persistent as $\left(b, d_{1}\right) \in$ $D_{2} \cup D_{3}$.

According to Figure 2, it is obvious that the range of $D_{1}$, in which the population is extinct, is enlarging as $\tau$ increases.

From Figure 2, for any given $d_{1}$ and $\tau$, when $b$ increases from zero, (4) first has no positive equilibrium and its trivial equilibrium is globally stable; when $b$ passes through the curve $l_{1}$, the trivial equilibrium is unstable, and the positive equilibrium appears and it is locally asymptotically stable; when it passes through the curve $l_{2}$ again, both the trivial and the positive equilibria are unstable. For any given $\tau$, if $0<$ $b \leq 1$, the trivial equilibrium is globally stable for an arbitrary $d_{1}$; if $1<b \leq \exp \left(1-\sec \omega_{1}\right)$, the locally asymptotically stable positive equilibrium could disappear as $d_{1}$ increases and passes through the curve $l_{1}$; if $b>\exp \left(1-\sec \omega_{1}\right)$, the stability of the positive equilibrium could change from unstable to stable, then to disappearing as $d_{1}$ increases and passes through the curves $l_{2}$ and $l_{1}$.

On the other hand, for the given $b$ and $d_{1}$, how does the value of $\tau$ affect the stability of equilibria $N=0$ and $N=N^{*}$ ?

From Theorems 2 and $4, N=0$ is globally stable if and only if $\tau \geq \ln b / d_{1} ; N=N^{*}$ is locally asymptotically stable if $0<(\ln b-2) / d_{1} \leq \tau<\ln b / d_{1}$. Then, how is the situation $0<\tau<(\ln b-2) / d_{1}$ ?

We define function $H(\omega):=\sec \omega+d_{1} \omega \cot \omega-(1-\ln b)$ for $\omega \in(\pi / 2, \pi)$. Straightforward computation yields

$$
\begin{gathered}
H^{\prime}(\omega)=\sec \omega \tan \omega+d_{1}\left(\cot \omega-\omega \csc ^{2} \omega\right), \\
H^{\prime \prime}(\omega)=\sec \omega\left(2 \tan ^{2} \omega+1\right)+2 d_{1} \csc ^{2} \omega(\omega \cot \omega-1)<0
\end{gathered}
$$

for $\omega \in(\pi / 2, \pi)$, where both $H(\omega)$ and $H^{\prime}(\omega)$ are continuous in the interval $(\pi / 2, \pi)$.

Note that

$$
\begin{gathered}
\lim _{\omega \rightarrow(\pi / 2)^{+}} H(\omega)=\lim _{\omega \rightarrow \pi^{-}} H(\omega)=-\infty \\
\lim _{\omega \rightarrow(\pi / 2)^{+}} H^{\prime}(\omega)=+\infty, \quad \lim _{\omega \rightarrow \pi^{-}} H^{\prime}(\omega)=-\infty
\end{gathered}
$$

then function $H(\omega)$ has a unique extreme point in the interval $(\pi / 2, \pi)$, denoted by $\bar{\omega}^{*}$, and its maximum is $H\left(\bar{\omega}^{*}\right)$.

We further discuss function $H(\omega)$ for $\omega \in(\pi / 2, \bar{\omega}) \subset$ $(\pi / 2,+\infty)$, where $\bar{\omega}$ is the root of equation $-(\ln b-2) / d_{1}=$ $\omega \cot \omega$ in the interval $(\pi / 2, \pi)$ for $\ln b>2$. Here, $H(\bar{\omega})=$ $\sec \bar{\omega}+1<0$. Therefore, with respect to sign of function $H(\omega)$ in $(\pi / 2, \bar{\omega})$, we have the following statements.

If $\bar{\omega} \leq \bar{\omega}^{*}, H(\omega)<0$ for $\omega \in(\pi / 2, \bar{\omega})$;

If $\bar{\omega}>\bar{\omega}^{*}$ and $H\left(\bar{\omega}^{*}\right)<0, H(\omega)<0$ for $\omega \in(\pi / 2, \bar{\omega})$;

If $\bar{\omega}>\bar{\omega}^{*}$ and $H\left(\bar{\omega}^{*}\right)>0, H(\omega)$ has exactly two zeros in the interval $(\pi / 2, \bar{\omega}), \omega_{1}^{*}$ and $\omega_{2}^{*}\left(\omega_{1}^{*}<\omega_{2}^{*}\right)$, such that $H(\omega)<0$ for $\omega \in\left(\pi / 2, \omega_{1}^{*}\right) \bigcup\left(\omega_{2}^{*}, \bar{\omega}\right)$ and $H(\omega)>$ 0 for $\left(\omega_{1}^{*}, \omega_{2}^{*}\right)$. 


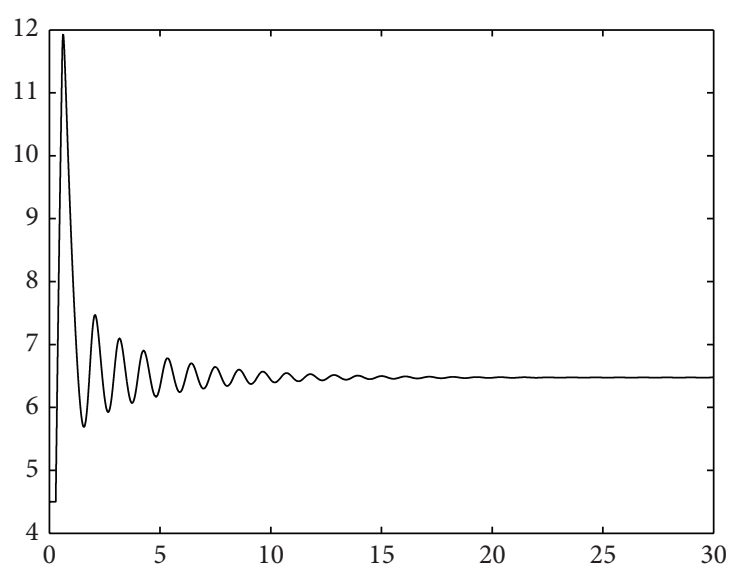

(a)

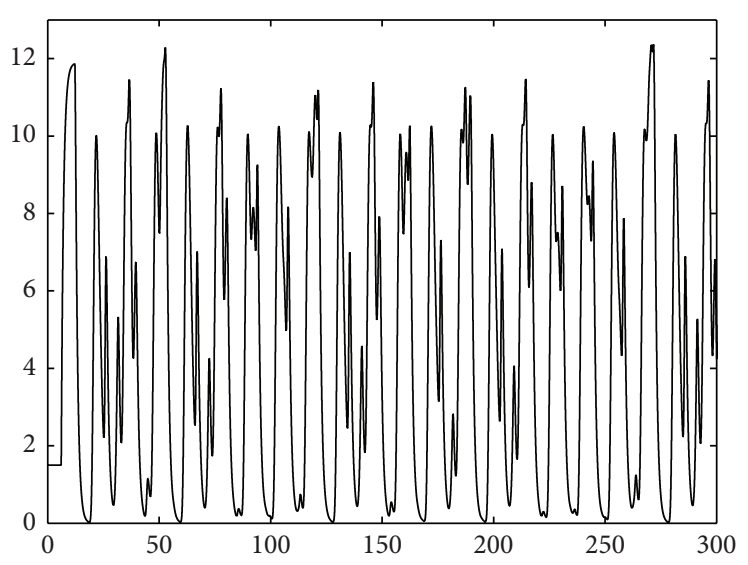

(c)

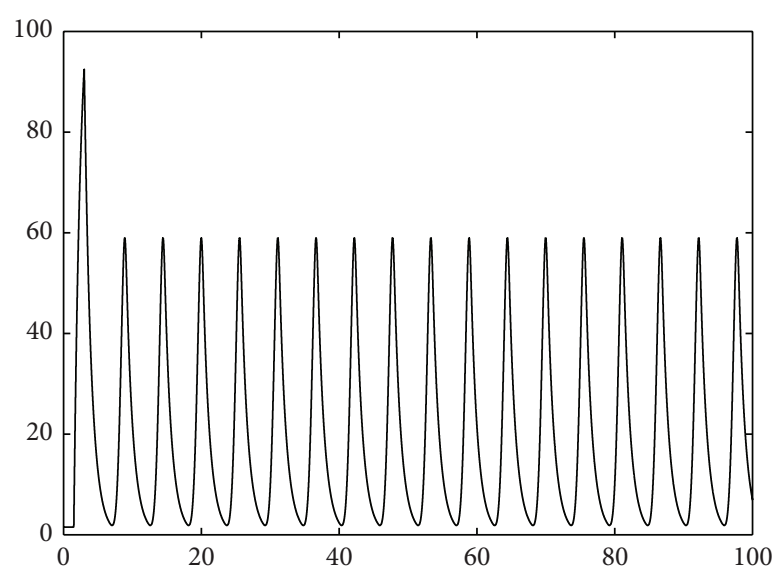

(b)

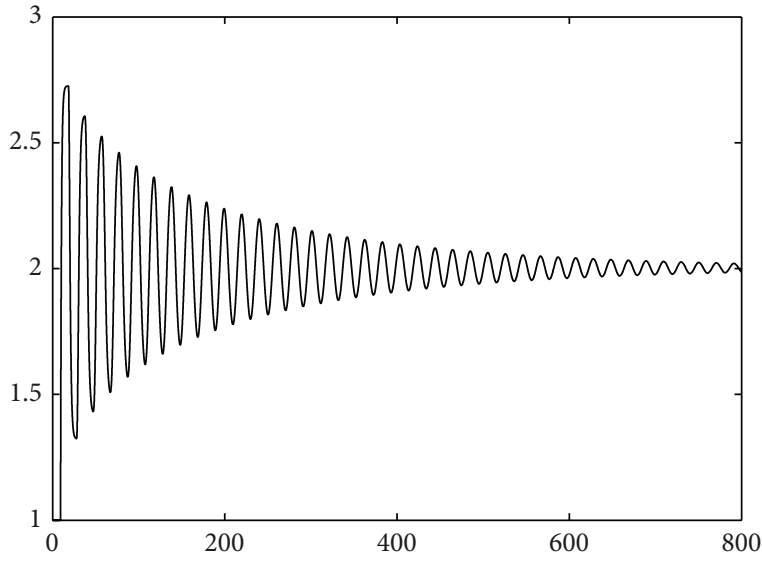

(d)

FIGURE 3: The solution curves of (4) with $b=750$ and $d_{1}=0.5$. Correspondingly, $(\ln b-2) / d_{1}=9.2401464, \tau_{1}^{*}=0.3271975$, and $\tau_{2}^{*}=$ 9.1459110. In (a), we choose $\tau=0.29<\tau_{1}^{*}$, then $N^{*}=6.47507321$, which is locally asymptotically stable. In (b), we choose $\tau=1.5 \in\left(\tau_{1}^{*}, \tau_{2}^{*}\right)$, then $N^{*}=5.87007321$ which is unstable, and there is a periodic solution for (4). In (c), we choose $\tau=6.10 \in\left(\tau_{1}^{*}, \tau_{2}^{*}\right)$, then $N^{*}=3.57007321$, and chaos occurs for (4). In (d), we choose $\tau=6.10>\tau_{2}^{*}$, then $N^{*}=2.00257321$, which is locally asymptotically stable.

For function $\omega=\omega(\tau) \in(\pi / 2, \pi)$ defined by (16) for $\tau \in(0,+\infty)$, it is continuous and increasing in the interval $(0,+\infty)$, since it follows from (16) that

$$
\frac{d \omega}{d \tau}=-\frac{1}{\cot \omega-\omega \csc ^{2} \omega}>0
$$

for $\omega \in(\pi / 2, \pi)$. And it is easy to know that $\lim _{\tau \rightarrow 0^{+}} \omega(\tau)=$ $\pi / 2$ and $\lim _{\tau \rightarrow+\infty} \omega(\tau)=\pi$. Further, according to the properties of function $\omega=\omega(\tau)$ and the definition of $\bar{\omega}$, for the given $b$ and $d_{1}, \omega(\tau)$ changes from $\pi / 2$ to $\bar{\omega}$ as $\tau$ increases from 0 to $(\ln b-2) / d_{1}$. Since $\cos \omega<0$ for $\omega \in(\pi / 2, \pi)$, the signs of functions $H(\omega)$ and $1-\left(1-d_{1} \omega \cot \omega-\ln b\right) \cos \omega$ are opposite. Notice that $\tau$ and $\omega(\tau)$ satisfy (16), then function $H(\omega)$ has the opposite sign to function $1-\left(1+d_{1} \tau-\ln b\right) \cos \omega$. Denoting the values of $\tau$ corresponding to $\omega_{1}^{*}$ and $\omega_{2}^{*}$ by $\tau_{1}^{*}$ and $\tau_{2}^{*}$, respectively, from Theorems 4 and 6 we have the following results.

Theorem 7. The positive equilibrium $N=N^{*}$ of (4) is locally asymptotically stable if one of the following conditions is satisfied: (i) $(\ln b-2) / d_{1} \leq \tau<\ln b / d_{1}$;

(ii) $\bar{\omega} \leq \bar{\omega}^{*}$ and $0<\tau<(\ln b-2) / d_{1}$;

(iii) $\bar{\omega}>\bar{\omega}^{*}, H\left(\bar{\omega}^{*}\right)<0$, and $0<\tau<(\ln b-2) / d_{1}$;

(iv) $\bar{\omega}>\bar{\omega}^{*}, H\left(\bar{\omega}^{*}\right)>0$, and $0<\tau<\tau_{1}^{*}$;

(v) $\bar{\omega}>\bar{\omega}^{*}, H\left(\bar{\omega}^{*}\right)>0$, and $\tau_{2}^{*}<\tau<(\ln b-2) / d_{1}$.

The positive equilibrium $N=N^{*}$ of (4) is unstable if $\bar{\omega}>$ $\bar{\omega}^{*}, H\left(\bar{\omega}^{*}\right)>0$ and $\tau_{1}^{*}<\tau<\tau_{2}^{*}$.

Additionally, the condition for the existence of the positive equilibrium $N=N^{*}$ is $\tau<\ln b / d_{1}$, so we can also give the other kinds of expressions with respect to Theorem 7 in the following.

Theorem 8. Only if the positive equilibrium $N=N^{*}$ of (4) exists, it is locally asymptotically stable when one of the following conditions is satisfied:

(i) $b \leq e^{2}$;

(ii) $b>e^{2}$ and $\bar{\omega} \leq \bar{\omega}^{*}$;

(iii) $b>e^{2}, \bar{\omega}>\bar{\omega}^{*}$, and $H\left(\bar{\omega}^{*}\right)<0$. 


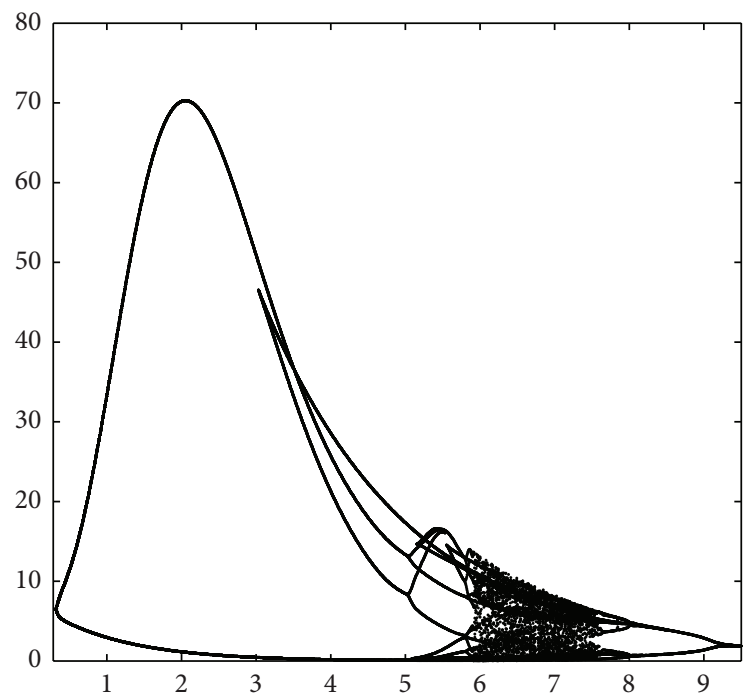

FIgURE 4: The bifurcation diagram of the parameter $\tau$ as $b=750$ and $d_{1}=0.5$.

When the positive equilibrium $N=N^{*}$ of (4) exists, with increasing of $\tau$ its stability can change from stable to unstable and to stable again when the conditions: $b>e^{2}, \bar{\omega}>\bar{\omega}^{*}$, and $H\left(\bar{\omega}^{*}\right)>0$ are satisfied. And the change occurs in turn as $\tau$ passes through $\tau_{1}^{*}$ and $\tau_{2}^{*}$.

Theorem 7 or 8 shows that the stability of the positive equilibrium $N=N^{*}$ of (4) does not change with variation of value of $\tau$ as one of the first three conditions in them is satisfied and that $N=N^{*}$ can undergo the stability switch with increasing of $\tau$ as $\bar{\omega}>\bar{\omega}^{*}$ and $H\left(\bar{\omega}^{*}\right)>0$. And the stability switch happens at $\tau=\tau_{1}^{*}$ and $\tau=\tau_{2}^{*}$. Correspondingly, it can be verified that the Hopf bifurcation also occurs at $\tau=\tau_{1}^{*}$ and $\tau=\tau_{2}^{*}$. Numerical simulations illustrate the existence of periodic solution and chaos as $\tau \in$ $\left(\tau_{1}^{*}, \tau_{2}^{*}\right)$ (Figure 3 ).

On the other hand, when the parameters $\tau, d_{1}$, and $b$ are used as the bifurcation parameter respectively, the associated parameter bifurcation diagrams are given in Figures 4, 5, and 6 , respectively. They may show the complexity of dynamic behaviors of model (5), including chaos.

\section{Conclusion}

In this paper, we first proved the positivity and the ultimate boundedness of model (1) and obtained the threshold determining the global stability of the trivial equilibrium and the existence of the positive equilibrium. Next, the stability of the positive equilibrium is investigated by means of Pontryagin's method, and the necessary and sufficient conditions ensuring the local stability of the positive equilibrium are obtained. Lastly, the dependence of stability of the positive equilibrium on the parameter values is analyzed, and the stability switch with variation of the maturation time is discussed completely. Additionally, numerical simulations exhibit that chaos may occur for certain parameter values and show that the local

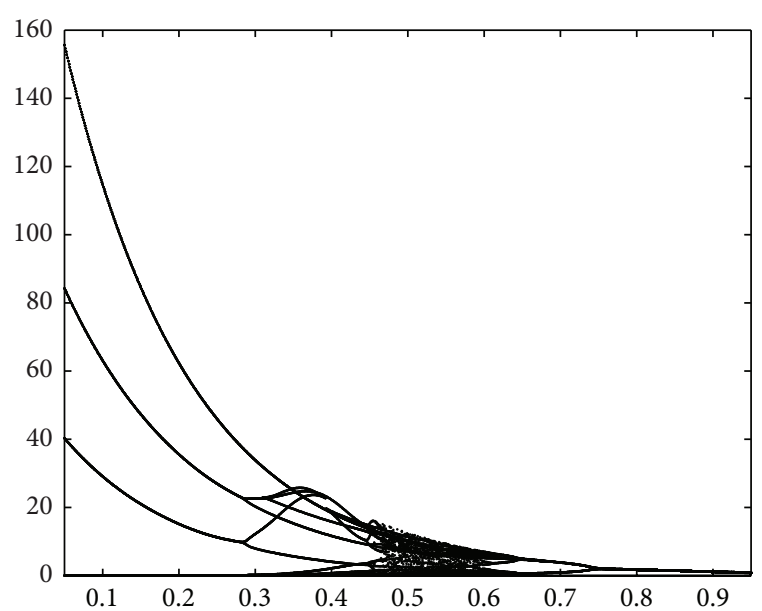

Figure 5: The bifurcation diagram of the parameter $d_{1}$ as $b=750$ and $\tau=6.1$.

asymptotical stability of the positive equilibrium implies its global stability. These results of numerical simulations need to be further proved rigorously. On the other hand, in order to show the dynamic complexity of (1), we also gave the associated parameter bifurcation diagram.

\section{Appendix}

We here introduce a method for determining when the roots of characteristic equation $P\left(z, e^{z}\right)=0$ are in the left halfplane, where $P(z, w)$ is a polynomial in $z, w$. The method is due to Pontryagin [10], which is referred to as the Pontryagin's method.

Suppose that $P(z, w)$ is a polynomial in $z, w$,

$$
P(z, w)=\sum_{m=0}^{r} \sum_{n=0}^{s} a_{m n} z^{m} w^{n},
$$

where $a_{r s} z^{r} w^{s}$ is called the principal term of the polynomial if $a_{r s} \neq 0$ and, if, for each other term $a_{m n} z^{m} w^{n}$ with $a_{m n} \neq 0$, we have either $r>m, s>n, r=m, s>n$, or $r>m, s=n$. Clearly, not every polynomial has a principal term.

Theorem 9. Let $\Delta(z)=P\left(z, e^{z}\right)$, where $P(z, w)$ is a polynomial with principal term. Suppose that $\Delta(i y), y \in \mathbb{R}$, is separated into its real and imaginary parts, $\Delta=F(y)+i G(y)$. If all zeros of $\Delta(z)$ have negative real parts, then the zeros of $F(y)$ and $G(y)$ are real, simple, and alternate and

$$
G^{\prime}(y) F(y)-G(y) F^{\prime}(y)>0
$$

for $y \in \mathbb{R}$. Conversely, all zeros of $\Delta(z)$ will be in the left half-plane provided that either of the following conditions is satisfied.

(i) All the zeros of $F(y)$ and $G(y)$ are real, simple, and alternate and inequality (A.2) is satisfied for at least one $y$.

(ii) All the zeros of $F(y)$ are real and, for each zero, inequality (A.2) is satisfied. 


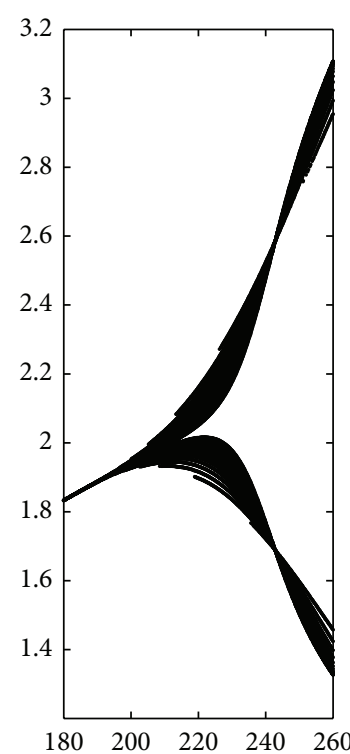

(a)

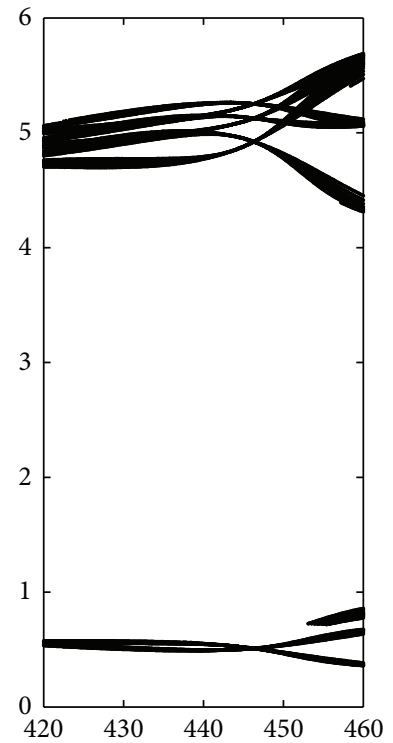

(b)

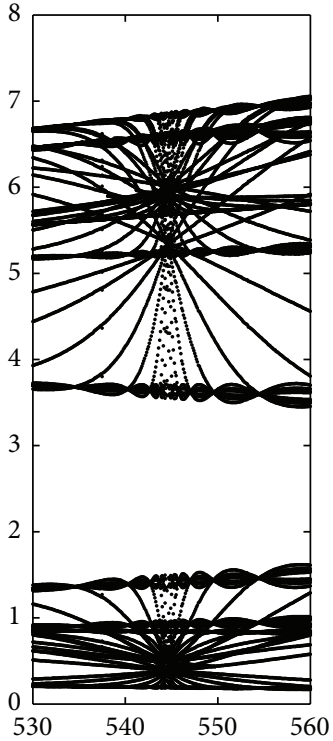

(c)

FiguRE 6: The bifurcation diagrams of the parameter $b$ as $d_{1}=0.6$ and $\tau=5.6$. Here, corresponding to Figures (a), (b), and (c), the values of $b$ are chosen in three intervals, [180,260], [420,470], and [530,560], respectively. From them we can see that the bifurcation features corresponding to the different intervals are distinct.

(iii) All the zeros of $G(y)$ are real and, for each zero, inequality (A.2) is satisfied.

On the other hand, in order to prove that all the zeros of $F(y)$ or $G(y)$ are real, we need the following results. Suppose that $f(z, u, v)$ is a polynomial in $z, u, v$ with real coefficients which has the form

$$
f(z, u, v)=\sum_{m=0}^{r} \sum_{n=0}^{s} z^{m} \phi_{m}^{(n)}(u, v),
$$

where $\phi_{m}^{(n)}(u, v)$ is a homogeneous polynomial of degree $n$ in $u, v$. The principal term in the polynomial $f(z, u, v)$ is defined as the term $z^{r} \phi_{r}^{(s)}(u, v)$ for which either $r>m, s>n, r=m$, $s>n$, or $r>m, s=n$ for all other terms in (A.3).

Let $z^{r} \phi_{r}^{(s)}(u, v)$ denote the principal term of $f(z, u, v)$ in (A.3); let $\phi_{*}^{(s)}(u, v)$ denote the coefficient of $z^{r}$ in $f(z, u, v)$,

$$
\phi_{*}^{(s)}(u, v)=\sum_{n=0}^{s} \phi_{r}^{(n)}(u, v)
$$

and let

$$
\Phi_{*}^{(s)}(z)=\phi_{*}^{(s)}(\cos z, \sin z) .
$$

Theorem 10. Let $f(z, u, v)$ be a polynomial with principal term $z^{r} \phi_{r}^{(s)}(u, v)$. If $\varepsilon$ is such that $\Phi_{*}^{(s)}(\varepsilon+i y) \neq 0, y \in \mathbb{R}$, then, for sufficiently large integers $k$, the function $F(z)=$ $f(z, \cos z, \sin z)$ will have exactly $4 k s+r$ zeros in the strip $-2 k \pi+\varepsilon \leq \operatorname{Re} z \leq 2 k \pi+\varepsilon$. Conversely, the function $F(z)$ will have only real roots if and only if, for sufficiently large integer $k$, it has exactly $4 k s+r$ roots in the strip $-2 k \pi+\varepsilon \leq \operatorname{Re} z \leq 2 k \pi+\varepsilon$.

\section{Conflict of Interests}

The authors declare that there is no conflict of interests regarding the publication of this paper.

\section{Acknowledgments}

This work is supported by the National Natural Science Fund of China (nos. 11371369 and 11371313).

\section{References}

[1] K. Cooke, P. van den Driessche, and X. Zou, "Interaction of maturation delay and nonlinear birth in population and epidemic models," Journal of Mathematical Biology, vol. 39, no. 4, pp. 332-352, 1999.

[2] X.-Q. Zhao and X. Zou, "Threshold dynamics in a delayed SIS epidemic model," Journal of Mathematical Analysis and Applications, vol. 257, no. 2, pp. 282-291, 2001.

[3] G. Fan, J. Liu, P. van den Driessche, J. Wu, and H. Zhu, "The impact of maturation delay of mosquitoes on the transmission of West Nile virus," Mathematical Biosciences, vol. 228, no. 2, pp. 119-126, 2010.

[4] K. L. Cooke, R. H. Elderkin, and W. Huang, "Predator-prey interactions with delays due to juvenile maturation," SIAM Journal on Applied Mathematics, vol. 66, no. 3, pp. 1050-1079, 2006.

[5] S. Ruan, "Delay differential equations in single species dynamics," in Delay Differential Equations and Applications, vol. 205 of NATO Sci. Ser. II Math. Phys. Chem., pp. 477-517, Springer, Dordrecht, The Netherlands, 2006.

[6] Z. Jiang and W. Zhang, "Bifurcation analysis in single-species population model with delay," Science China. Mathematics, vol. 53, no. 6, pp. 1475-1481, 2010. 
[7] E. Beretta and Y. Kuang, "Geometric stability switch criteria in delay differential systems with delay dependent parameters," SIAM Journal on Mathematical Analysis, vol. 33, no. 5, pp. 1144$1165,2002$.

[8] J. Wei and X. Zou, "Bifurcation analysis of a population model and the resulting SIS epidemic model with delay," Journal of Computational and Applied Mathematics, vol. 197, no. 1, pp. 169187, 2006.

[9] J. P. LaSalle, The Stability of Dynamical Systems, SIAM, Philadelphia, Pa, USA, 1976.

[10] J. Hale, Theory of Functional Differential Equations, vol. 3 of Applied Mathematical Sciences, Springer, New York, NY, USA, 1993. 


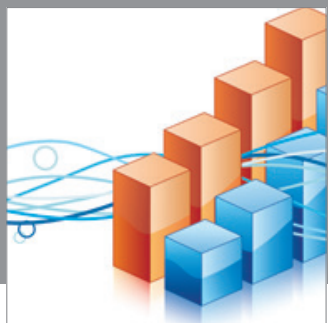

Advances in

Operations Research

mansans

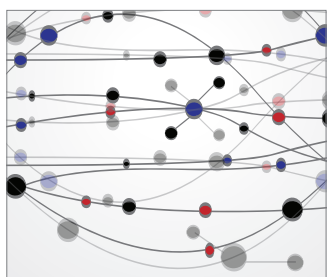

The Scientific World Journal
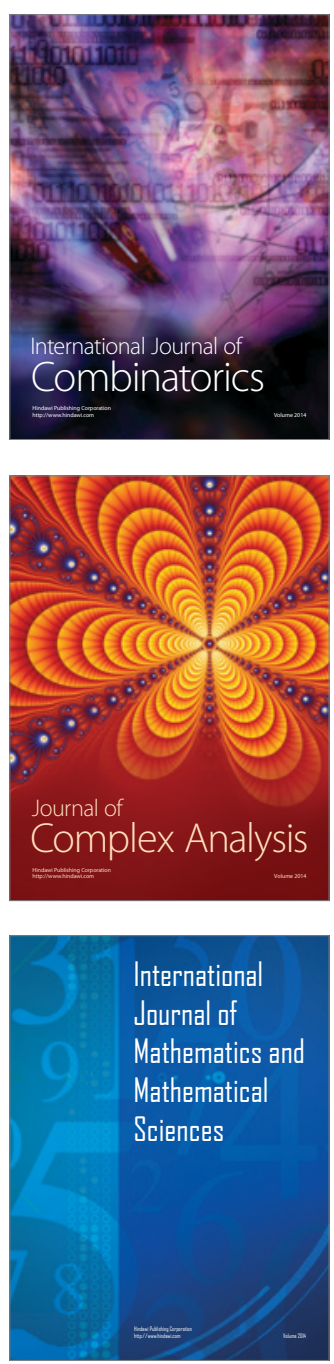
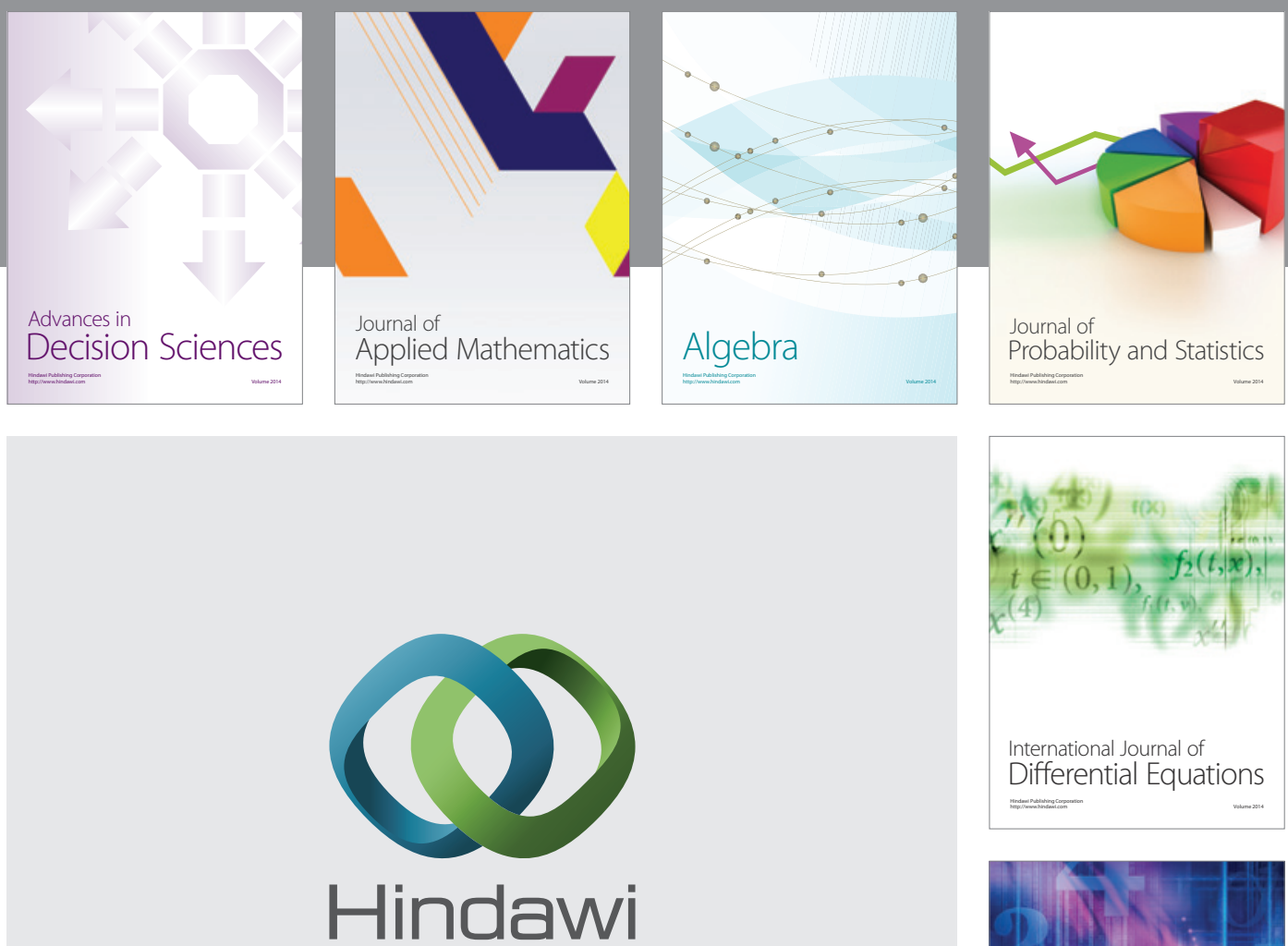

Submit your manuscripts at http://www.hindawi.com
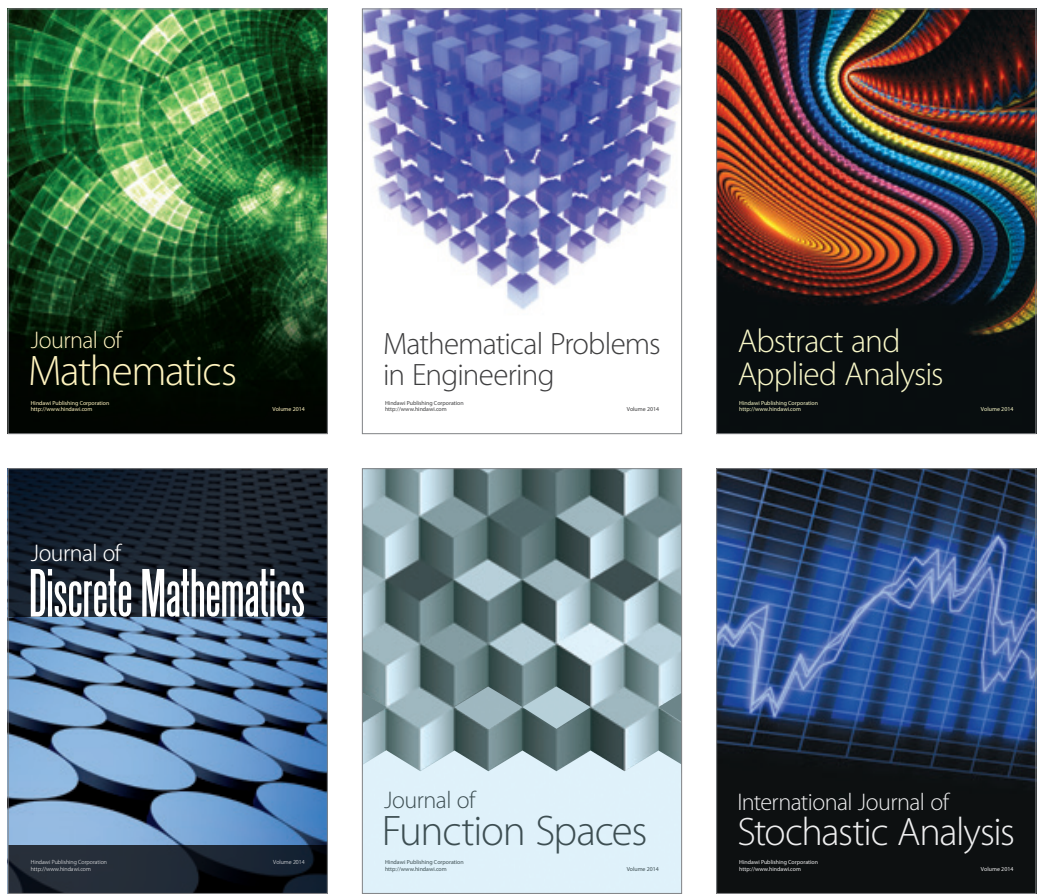

Journal of

Function Spaces

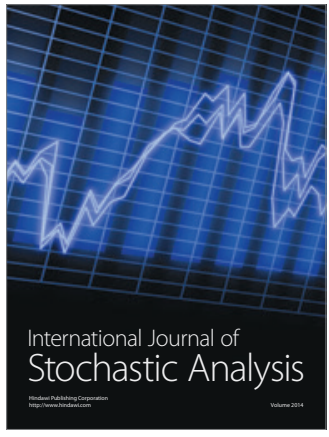

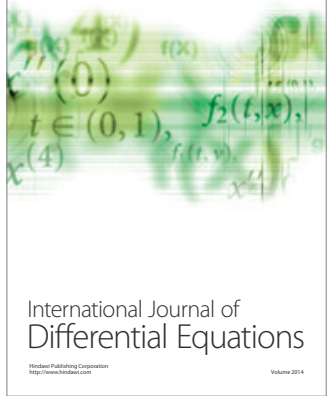
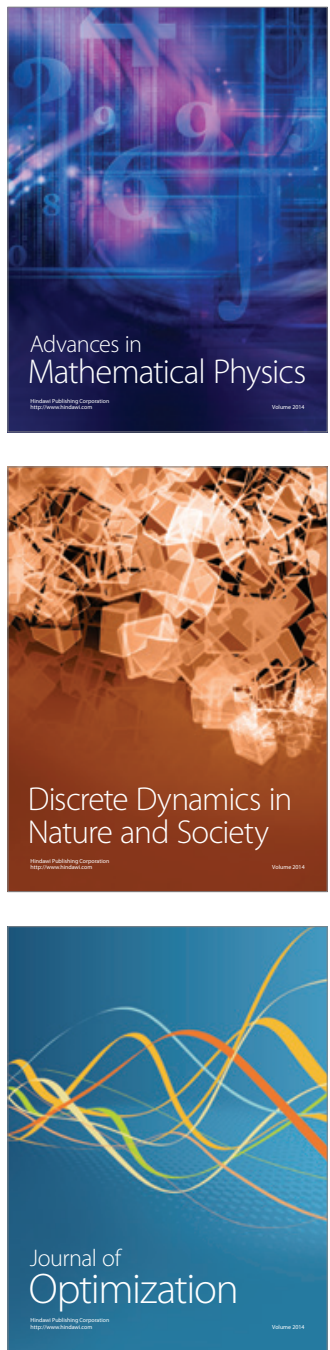